\title{
UTILIZAÇÃO DE LEITOS HOSPITALARES GERAIS EM RIBEIRÃO PRETO, SÃO PAULO (BRASIL) *
}

Yazlle Rocha, J. S. - Utilização de leitos hospitalares gerais em Ribeirão Preto, São Paulo (Brasil). Rev. Saúde públ.. S. Paulo, 9:477-93, 1975.

Resumo: Foi estudada a demanda de leitos hospitalares gerais do municipio de Ribeirão Preto, São Paulo (Brasil), no ano de 1972, pela população do municipio, a partir da utilização destes leitos. Valeu-se, para tanto, de um centro de informática de dados hospitalares. As caracteristicas da demanda encontrada foram: elevado coeficiente de internação, baixo indice de hospitalização, curta duração média das internações (determinada por fatores demográficos e do sistema previdenciário de internaçōes); utilização do indice de 2,43 leitos gerais 1.000 hab., naquele ano.

Unitermos: Hospitalização, demanda. Leitos hospitalares. Assistência médico-hospitalar.

\section{$1-$ INTRODUCAO}

Todos os avanços médicos nos últimos tempos pouco significam se não estendermos os seus benefícios a toda população. Este é o conceito moderno de assistência médica: "é o conjunto de meios diretos e específicos destinados a por ao alcance do maior número de indivíduos e de suas famílias, os recursos do diagnóstico precoce, tratamento oportuno, completo e restaurador e do seguimento posterior" (OPAS, 1962) 2.2.

Por outro lado, os próprios avanços médicos e a complexidade inerente a certos tipos de serviços médicos, têm tornado a assistência médica cada dia mais custosa agravando os problemas de governos com minguados recursos para investir no de- senvolvimento econômico e social dos povos. Mas ninguém discute atualmente se a Saúde (e a atenção médica) é elemento importante e se deve articular-se no esforço geral da nação, para o desenvolvimento.

Disto surge a necessidade de planejar o investimento de recursos, procurando métodos de maior alcance e rendimento, de racionalizar os instrumentos da assistência médica, evitar a sub-utilização e o desperdício, atendendo as necessidades mais prementes da população.

O hospital moderno desempenha importante papel nos programas de saúde e bem-estar social; seu objetivo fundamental é a prestação de atenção médica integral à comunidade.

* Parte da tese de Doutoramento apresentada à Faculdade de Medicina de Ribeirão Preto da USP, 1974.

* Do Departamento de Medicina Social da Faculdade de Medicina de Ribeirão Preto da USP - Caixa Postal 301 - 14100 - Ribeirão Preto, SP - Brasil 
YAZLLE RoCHA, J. S. - Utilização de leitos hospitalares gerais em Ribeirão Preto, São Paulo (Brasil). Rev. Saúde pribl., S. Paulo, 9:477-93, 1975.

Segundo a OPAS (1967) ${ }^{23}$ a ação do hospital deve: "ser integral (preventiva, curativa e de reabilitação); ser coordenada em nível local, regional e nacional; oferecer cuidado progressivo do paciente, isto é, de acordo às necessidades e condições clinicas do paciente (através de serviço ambulatorial, domiciliar ou institucional); e finalmente prestar-se à docência médica e para-médica".

No nosso trabalho incluímos como hospitais gerais, de acordo com Llewelyn-Davies e Macaulay ${ }^{16}$, 1969, hospitais que contam com serviços especializados e que atendem homens, mulheres e crianças, deles estando excluídos os destinados a doenças mentais e a tuberculose.

\section{2 - Necessidade, demanda, utilização}

O planejamento da inversão de recursos para a saúde pode se fazer de acordo com critérios como "necessidades" da comunidade, "demanda" expressa pela comunidade, ou em base à "utilização" de recursos que a comunidade vem efetuando nos últimos períodos.

A necessidade é obtida pela pesquisa da morbidade e mortalidade prevalentes na comunidade (podendo elas serem percebidas ou não pela população). A conversão de dados de morbidade e mortalidade em medidas de recursos de saúde envolve necessariamente a opinião de técnicos no assunto, implicando desta forma critérios subjetivos.

A demanda, entendida como a procura efetiva de serviços de saúde, por uma determinada população, pode ser expressa objetivamente e assim traduzida em recursos para a saúde. A demanda não é expressão de todas as necessidades de saúde da comunidade e pode até haver demanda sem "necessidade".

A utilização efetiva dos serviços ocorre por conta da demanda, podendo existir uma parte da demanda que não é satisfeita.

O esquema proposto por Anderson ${ }^{1}$ (1973), e por nós modificado, expressa a relação entre estes elementos (Fig. 1).

Figura 1

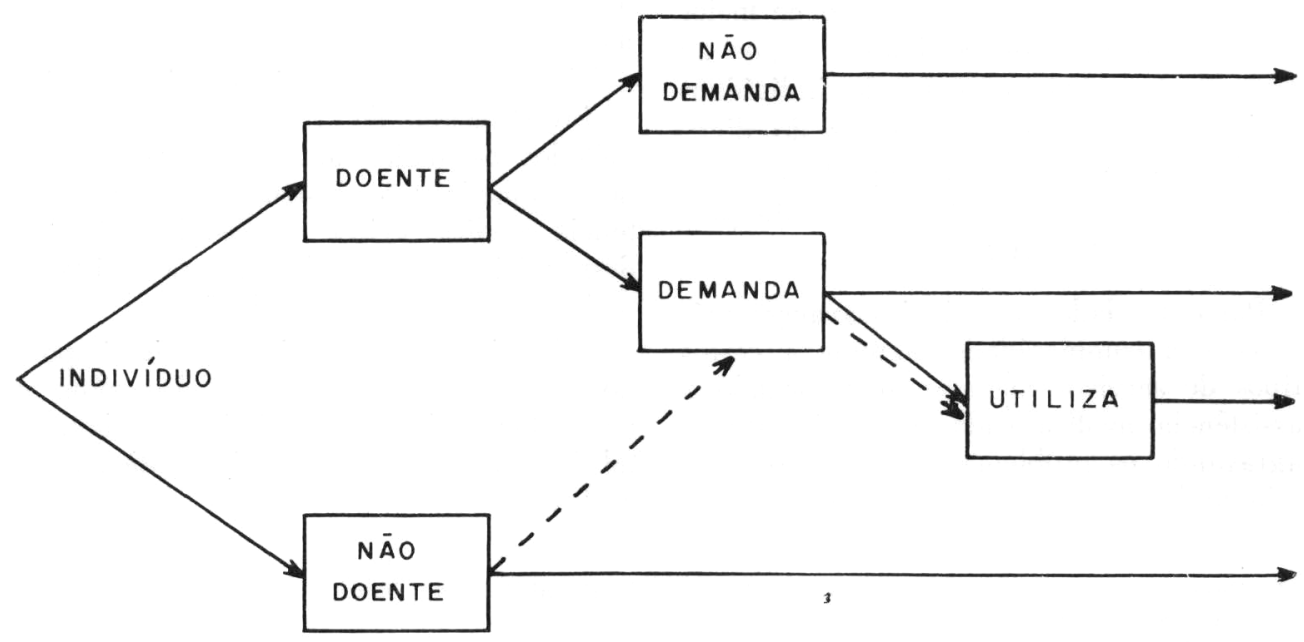

Fig. 1 - Necessidade, demanda e utilização. 
YAZLLE ROCHA, J. S. - Utilização de leitos hospitalares gerais em Ribeirão Preto, São Paulo (Brasil). Rev. Saúde públ., S. Paulo, 9:477-93, 1975.

A utilização dos recursos pode ser dirigida em um ou outro sentido pelo sistema de atenção médica vigente, concordando ou não com a tendência da demanda na forma expressa pela comunidade.

Ao se definir a utilização de serviços de saúde por uma comunidade deve se levar em conta a sub-utilização ("underuse") e a super-utilização ("overuse"), no sentido da necessidade ou demanda não coberta, havendo recursos disponíveis no primeiro caso, e da utilização por conta de uma demanda sem necessidade no segundo caso.

Llewelyn-Davies e Macaulay ${ }^{16}$ (1969) referem os seguintes fatores envolvidos na utilização dos leitos hospitalares:

- disponibilidade de leitos hospitalares

- sistemas de pagamento dos serviços hospitalares

- tipo de população e sua distribuição por idade e sexo

- distribuição geográfica dos leitos

- disponibilidade de serviços de assistência médica fora do hospital

- capacidade dos serviços auxiliares do hospital

- hábitos médicos e normas sociais

- número de médicos

- investigação e ensino no hospital

- hospitais particulares

- tipos de moradia

- morbidade

- organização interna do hospital

Como vemos, muitos fatores estão envolvidos na utilização de leitos hospitalares, o que torna muito difícil a previsão da utilização futura dos leitos. A distribuição adequada dos recursos para assistência médica, de forma a atender a demanda e necessidades futuras, é meta muito importante dentro do planejamento da assistência médica, de um país.
1.3 - Métodos de investigação

Navarro ${ }^{19}$ (1969) discute 6 métodos para o planejamento da distribuição de serviços de saúde individuais:

I - Método baseado na mortalidade

II - Método baseado na morbidade

III - Métodos baseados na utilização

IV - Métodos baseados na distribuição

V - Método baseado no desempenho do sistema

VI - Método baseado na estrutura do sistema

Métodos baseados na utilização (III): nestes métodos o uso presente dos recursos é tomado como estimador do uso futuro. Baseiam-se no conceito de demanda que é mais objetivo, porém exigem que se satisfaçam duas condições: os recursos devem ser adequados, isto é, dispor-se de facilidades suficientes para encontrar a demanda; é preciso também uma boa coordenação e distribuição entre os recursos (relações funcionais entre os recursos e a população) (Brooks e col. ${ }^{3}, 1964$; Feldstein e German ?, 1965; Burkens ${ }^{4}$, 1966).

Englobam-se neles os seguintes métodos:

Método baseado na demanda: consiste em extrapolar a presente razão recursos/população (que é condicionada pela demanda), a uma projeção futura da população. Este método somente leva em conta o aumento da demanda decorrente do crescimento demográfico.

Este método é criticável, segundo $\mathrm{Na}$ varro ${ }^{19}$, porque não só mantém o "status-quo" da razão atual recursos/população, mas também aumenta os defeitos que ela possa ter ao ser extrapolada ao futuro

Métodos baseados na distribuição (IV): referem-se às relações geográficas e funcionais entre os recursos e a população servida (Newell ${ }^{21}$, 1964). Englobam-se aqui os dois métodos seguintes: 
YAZLLE ROCHA, J. S. - Etilização de leitos hospitalares gerais em Ribeirão Preto, São Paulo (Brasil). Rev. Saúde públ., S. Paulo, 9:477-93, 1975.

a) Método centrado nas facilidades: consiste em pesquisar um grupo de hospitais. definindo a população por eles servida. Atrarés de um estudo dos egressos de cada hospital se conhece o total de pacientes provenientes de determinada área e sua proporção do total de pacientes atendidos pelo hospital. Somando-se estas partes de cada hospital, é possível conhecer o total de pacientes proveniente duma determinada área, atendidos pelo conjunto de hospitais em dado período. Projetando-se a mudança futura da população. estima-se a necessidade que precisará ser atendida. Como crítica observa-se que este método não leva em conta os fatores de escolha de um determinado hospital pela população da área.

b) Método centrado na população: baseia-se na análise do padrão de utilização do hospital por uma população definida. Mais do que a demanda, mede o uso atual dos leitos da área ou adjacências. Tem a vantagem de enfocar a assistência a uma comunidade determinada, da qual a atenção hospitalar é um dos principais serviços de saúde mas não é o único.

\section{4 - Estudos e recomendações no Exterior e no Brasil}

A OPAS ${ }^{22}$ (1962) recomenda para os países latino-americanos como um índice prudente, 4.5 leitos $/ 1.000$ hah.. destacando que não é possivel fixar-se em um número sabendo que as necessidades variam de acordo a múltiplos fatores. embora não justifique o indice recomendado.

Flisfisch ${ }^{8}$ (1966) reitera a estimativa de 4.5 leitos por 1.000 habitantes para pacientes agudos. assinalando o grande déficit de leitos existentes nos países da área.

San Martin ${ }^{27}$ (1968) propõe de maneira geral o índice de 4.5 leitos gerais/ 1.000 hab. baseado em que $10 \%$ da po- pulação ingressa ao hospital ao menos uma rez ao ano, com uma duração média da internação de 14 dias e sendo cada leito utilizado 325 dias por ano, teremos que 4.5 leitos/1.000 habitantes serão suficientes para atender a essa população.

No Brasil temos as seguintes recomendações:

- Pedroso ${ }^{25}$ (1954): 4.6 leitos gerais 1.600 hab.

- Casella ${ }^{5}$ (1967): centros principais 6.5 leitos 1.000 hab.;

centros secundários 5.0 leitos $/ 1.000$ hab.:

distritos isolados 2.5 leitos/1.000 hab.

- Mello ${ }^{15}$ (1969): 4.5 - 5.5 leitos/1.000 hab. para hospitalização geral mais 2 leitos/1.000 hab. para crônicos

- Yunes ${ }^{33}$ (1970): extensão da rede metropolitana de São Paulo para atingir

5.0 leitos $/ 1.000$ hab. a médio prazo 7.0 leitos $/ 1.000$ hab. a longo prazo

- Martins ${ }^{1 i}$ (1971): $4.5-5.0$ leitos 1.000 hab.

- Gonçalves e col. ${ }^{12}$ (1972): 4.0 leitos/1.000 hab. para clínica médica e cirurgia

1.5 leitos $/ 1.000$ hab. para obstetrícia 1.0 leitos/ 1.000 hab. para pediatria

As razões leitos gerais/habitantes. preconizadas por autores nacionais. têm-se fundamentado principalmente em dados de outros paises e no empirismo de alguns serviços existentes no Brasil; faltam trahalhos que se proponham quantificar as necessidades de recursos para a atenção médico-hospitalar de uma dada população brasileira seguindo qualquer dos métodos antes apresentados. Acreditamos que a 
YAZLLE ROCHA, J. S. - Utılização de leitos hospitalares gerais em Ribeirão Preto, São Paulo (Brasil). Rer. Saúde públ., S. Paulo, 9:477-93, 1975.

principal razão de carência de estudos desta natureza repouse na ausência de estatísticas, principalmente hospitalares.

Va cidade de Ribeirão Preto, como fruto do trahalho desenvolvido por uma equipe. liderada por um docente da Faculdade de Medicina de Ribeirão Preto, criaram-se condiçōes que nos permitiram elahorar o presente estudo.

\section{$1.5-$ Objetiros}

Xosso estudo tem os seguintes objetivos:

- estudar a utilização de leitos hospitalares gerais. pela população do município de Ribeirão Preto. no ano de 1972:

- inferir a demanda da atenção médico-hospitalar geral da população de Ribeirão Preto no ano de 1972. atrarés do estudo da utilização.

\section{2 - MATERIAL E METODOS}

Atualmente. Ribeirão Preto constitui importante centro administrativo, comercial e educacional lcom numerosos cursos de nível médio. superior e técnicos). além de centro médico-hospitalar regional. O crescimento e desenvolvimento de Riberião Preto como importante centro médico regional. apesar dos estímulos recebidos nesse sentido, não obedeceu a um planejamento para a região ou estado. 0 crescimento da demanda de assistência médica no município deve ter sido o principal fator de impulso à concentração de recursos para a saúde neste local. Importante papel. neste sentido. deve ter exercido a Faculdade de Medicina local, e o Hospital das Clínicas da Faculdade de Medicina de Ribeirão Preto. ambos de renome nacional e internacional, aumentando a capacidade e elevando o padrão de assistência médica à cidade e região. Na cidade existe um centro de processamento de informações que cobre todos hospitais gerais da cidade. sendo esta a razão principal. além de certas características da cidade. fundamentais para o nosso trabalho. que nos fizeram escolher o município de Ribeirão Preto como área de nosso estudo.

O material utilizado neste trabalho consiste de informações sobre os egressos dos pacientes internados em hospitais gerais, dados populacionais e facilidades existentes para a atenção médica-hospitalar. no município de Ribeirão Preto, no ano de 1972.

Estão incluidas todas as internações em hospitais gerais de Ribeirão Preto, entre 1. de janeiro a 31 de dezembro de 1972. De cada internação foram estudados: sexo. idade, munićpípio de residência, categoria da internação (isto é. a fonte financiadora da hospitalização), data da internação. diagnóstico principal idefinido como a causa da internação). e secundários (outros diagnósticos). duração da internação e condição de saída (vivo ou morto). Estas informaçôes foram colhidas da folha de egresso dos pacientes, sendo que os dados de identificação do paciente são preenchidos pela funcionária do hospital encarregada da admissão. a partir das informaçóes e documentos apresentados pelo paciente, familiares ou responsáreis pelo mesmo; os dados médicos são preenchidos pelo médico responsável no momento do egresso.

A população mediana do município em 1972 foi estimada a partir dos censos demográficos de 1960 e de 1970 (Fundação IBGE ${ }^{9}, 1971$ ), baseando-nos no crescimento aritmético da população. Para obtermos a distribuição da população segundo os grupos etários desejados, utilizamos como estimativa a pirâmide populacional do Estado de São Paulo (Fundação IBGE ${ }^{11}, 1972$ ).

Algumas facilidades para a atenção médica, como: quantidade de leitos. sua localização geográfica. acessibilidade aos leitos hospitalares pela população, núme- 
YAZLLE RochA. J. S. - Utilizaçāo de leitos hospitalares gerais em Ribeirão Preto, São Paulo (Brasi1). Rev. Satide públ., S. Paulo, 9:477-93, 1975.

ro de médicos e ambulatórios foram estudados e também alguns indicadores do nível de saúde e das condiçōes sanitárias da população de Ribeirão Preto.

Nosso estudo está centrado na utilização dos leitos hospitalares gerais pela população do município de Ribeirão Preto, satisfazendo ao mesmo tempo as condições dos métodos haseados na utilização e na distribuição dos recursos (Navarro ${ }^{19}$, 1969).

Para o estudo da utilização dos leitos hospitalares gerais nos valemos da infra-estrutura e rotinas implantadas pelo Centro de Processamento de Dados Hospitalares $(\mathrm{CPDH})$, do Departamento de Medicina Social (DMS), da Faculdade de Medicina de Ribeirão Preto, neste município (Fávero e col. ${ }^{6}$. 1973).

Tendo iniciado suas atividades no campo em 1970, a CPDH rai mantendo e aperfeiçoando sua estrutura ao mesmo tempo que estende sua ação a outras cidades do Estado de São Paulo e de outros Estados.

\section{1 - Sistema de informação}

O CPDH obtém fundamentalmente dois tipos de informações: a) dados relativos ao paciente através de uma folha de egresso; b) dados relativos à utilização das enfermarias. através de uma folha de censo diário. Ao organizar o sistema de registro, colheita e processamento das informações, o CPDH valeu-se de experiências de centros estrangeiros. adaptando-as de acordo às condições locais. O sistema compreende 3 fases: a primeira, de registro e colheita das informações nos estabelecimentos hospitalares; a segunda, de elaboração destas informações (revisão, codificação e perfuração dos dados); e a terceira, do processamento eletrônico dos dados e sua publicação.

Neste trabalho são consideradas as informaçöes dos 7 hospitais gerais de Ribeirão Preto. todos convenentes com o CPDH do DMS:
Hospital dos Acidentados (HA)

- Hospital das Clínicas da Faculdade de Medicina de Ribeirão Preto (HC)

- Hospital São Francisco (HSF)

- Hospital São Lucas (HSL)

- Instituto Santa Lydia (ISL)

- Hospital Sociedade Portuguesa de Beneficência (HB)

- Santa Casa de Misericórdia de Ribeirão Preto (SC)

\section{2 - Cuidados especiais para o presente estudo}

Durante o processamento das informações relativas a este estudo, dedicamo-nos mais intensamente à supervisão das diferentes fases. Antes do processamento eletrônico para o nosso trabalho, os cartões foram testados quanto à congruência das informações (principalmente através do confronto da idade e sexo com os diagnósticos).

Tiremos especial cuidado durante este estudo de evitar a perda quantitativa das informaçóes. O CPDH confronta o número de altas recebidas com as informadas no censo diário de cada enfermaria, detectando assim toda vez que ocorre um egresso sem que tenha sido remetida a folha correspondente ao CPDH. Nestes casos um funcionário do CPDH foi sempre ao hospital para obter a folha que faltava. recuperando assim a informação.

Com o objetivo de avaliar a consistência das informações (entendida como a percentagem da concordância entre as informações contidas no cartão perfurado e as do prontuário médico), referidas ao CPDH. tomamos uma amostra casual simples. de cartões perfurados, de tamanho entre 5 e $10 \%$ do total de internaçôes de cada hospital no ano de 1972. Foram cotejadas as informações (do cartão) mais utilizadas neste estudo: idade. sexo. diagnósticos procedência do paciente, condição de saída. com as informações contidas no prontuário médico. 
YAZLLE RoCHA, J. S. - Utilização de leitus hospitalares gerais em Ribeirão Preto, São Paulo (Brasil). Rev. Saúde públ., S. Paulo, 9:477-93, 1975.

Para o processamento das informaçōes necessárias ao nosso estudo, mantivemos entendimento com o Centro de Processamento de Dados (CPD) da Escola de Engenharia de São Carlos, visando a elaboração do programa para processamento no equipamento IBM 1130 daquele estabelecimento.

Uma vez consolidados os programas procedeu-se ao processamento das informações.

\section{$3-$ RESULADOS}

\section{1 - Alguns fatores ligados à utilização de leitos hospitulares}

De acordo com nossa estimativa, a população do município de Ribeirão Preto em $1 .{ }^{\circ}$ de julho de 1972 era de 230.330 pessoas: $34.50 \%$ da população esteve entre 0 a 15 anos de idade; 52,20\% estava entre 15 e 50 anos; e 13,30\% estava acima de 50 anos de idade. $\mathrm{Na}$ cidade de Ribeirão Preto moram 90,60\% da população do município; $92,60 \%$ da população moram em zona urbana (zonas urbanas dos distritos de Ribeirão Preto, Bonfim Paulista e Guatapará) e apenas $7,40 \%$ moram na zona rural.

De acordo com Puffer e Serrano ${ }^{26}$ (1973) a natalidade na cidade de Ribeição Preto, no período julho-1968 a junho-1970, foi de 25.6 nascimentos/ 1.000 hab.

A mortalidade geral no município em 1972 foi de 7.9 óbitos/ 1.000 hab. (6. ${ }^{\mathrm{a}}$ Divisão Regional de Saúde, 1973 *). No período julho-1968 a junho-1970, a mortalidade infantil na cidade foi de 43 óbitos $/ 1.000$ nascidos vivos; a mortalidade neonatal no mesmo período foi 24,0 óbitos 1.000 nascidos vivos (Puffer-Serrano ${ }^{26}, 1973$ ).

A mortalidade proporcional de 50 anos ou mais (indicador Swaroop-Uemura), em

* Comunicação pessoal.
1972 foi $55.23 \%$ (6. Divisão Regional de Saúde *, 1973).

o município contava em 1972 com 45.530 prédios na zona urbana (Secretaria de Estado da Saúde $\left.{ }^{28}, 1973\right), 96.88 \%$ dos quais estavam ligados à rede pública de água. No distrito de Ribeirão Preto, praticamente a totalidade da água distribuída na rede pública provém de lençol profundo (nos outros dois distritos a água provém de lençol superficial). Estavam ligados à rede pública de esgoto, $80,70 \%$ dos prédios do município. A população da cidade era servida por eficiente serviço de colheita de lixo e a Prefeitura Municipal mantinha uma usina de industrialização do lixo. A Prefeitura gastou naquele ano quase $3 \%$ do seu orçamento com limpeza pública, colheita e tratamento do lixo (o orçamento total foi de Cr\$ 42.160 .450 .00 ).

Em 1972 havia no município 619 médicos (dando o índice de um médico para 372 habitantes), 347 dentistas, 88 enfermeiras e 133 farmacêuticos. $0 \mathrm{mu}$ nicípio contava com 12 hospitais ( 7 gerais e 5 especializados) e um pronto-socorro municipal. Os 7 hospitais gerais tinham ao todo 1.209 leitos e 100 berços. Do total de leitos. 226 eram exclusivamente para pediatria e 116 para obstetrícia. Os leitos restantes, na sua maioria, não se destinavam a um uso exclusivo, variando a utilização segundo a demanda. Estes leitos dão os seguintes índices: 5.25 leitos gerais/1.000 hab., 0,98 leitos de pediatria/ 1.000 hab. e 0.50 leitos de obsteritcia/1.000 hab. Contava também com 18 ambulatórios médicos (excluídos aqueles de hospitais), 82 farmácias e 7 laboratórios clínicos. Os 7 hospitais gerais dispõem-se em torno do centro comercial da cidade. Em cada bairro da cidade funciona ao menos um ambulatório médico pertencente à Prefeitura, rede de saúde pública do Estado ou Faculdade de Medicina local. Na sede dos outros dois distritos do município funcionam ambulatórios mantidos pela Prefeitura e Centro de Saúde local; na cidade existe 
YAZlLE ROCHA, J. S. - Utilização de leitos hospitalares gerais em Ribeirão Preto, São Paulo (Brasil). Rev. Saúde públ., S. Paulo, 9:477-93, 1975.

em funcionamento um serviço de ônibus urbano que atende a todos os bairros e a Prefeitura local possui 9 ambulâncias que atendem à população da cidade e dos distritos.

\section{2 - Utilização dos leitos hospitalares gerais}

Dos 442.494 leitos-dia de hospitais gerais disponíveis no ano de $1972,312.889$ foram utilizados dando $70,71 \%$ de ocupação destes leitos. Nos 7 hospitais a percentagem de ocupação naquele ano variou de $41 \%$ a $81 \%$ de ocupação (somente 2 hospitais tiveram ocupação maior que a média, um com $72 \%$ e outro com $81 \%)$.
Dos leitos-dia ocupados, $46,36 \%$ foram utilizados por pacientes do município de Ribeirão Preto: $32,32 \%$ por pacientes da Região de Ribeirão Preto e 21,31\% por pacientes de fora da Região de Ribeirão Preto. Esta utilização ocorreu por 40.832 internações (excluídos os recém-nascidos), sendo $28.234,(69,15 \%)$ de pacientes procedentes de Ribeirão Preto, $9.755(23,89 \%)$ de pacientes da Região de Ribeirão Preto e $2.843(6,96 \%)$ de pacientes de fora da 6 . $^{2}$ Região Administrativa. A duração média das internações de Ribeirão Preto (5,14 dias) foi a metade e um quarto respectivamente, da apresentada pelas internações da região de Ribeirão Preto e de fora da $6{ }^{a}$ Região Administrativa.

T A B E A 1

Distribuição dos pacientes internados e coeficientes de internação (por 1.000 habitantes) em hospitais gerais, da população de Ribeirão Preto, segundo grupo etário e sexo, em 1972

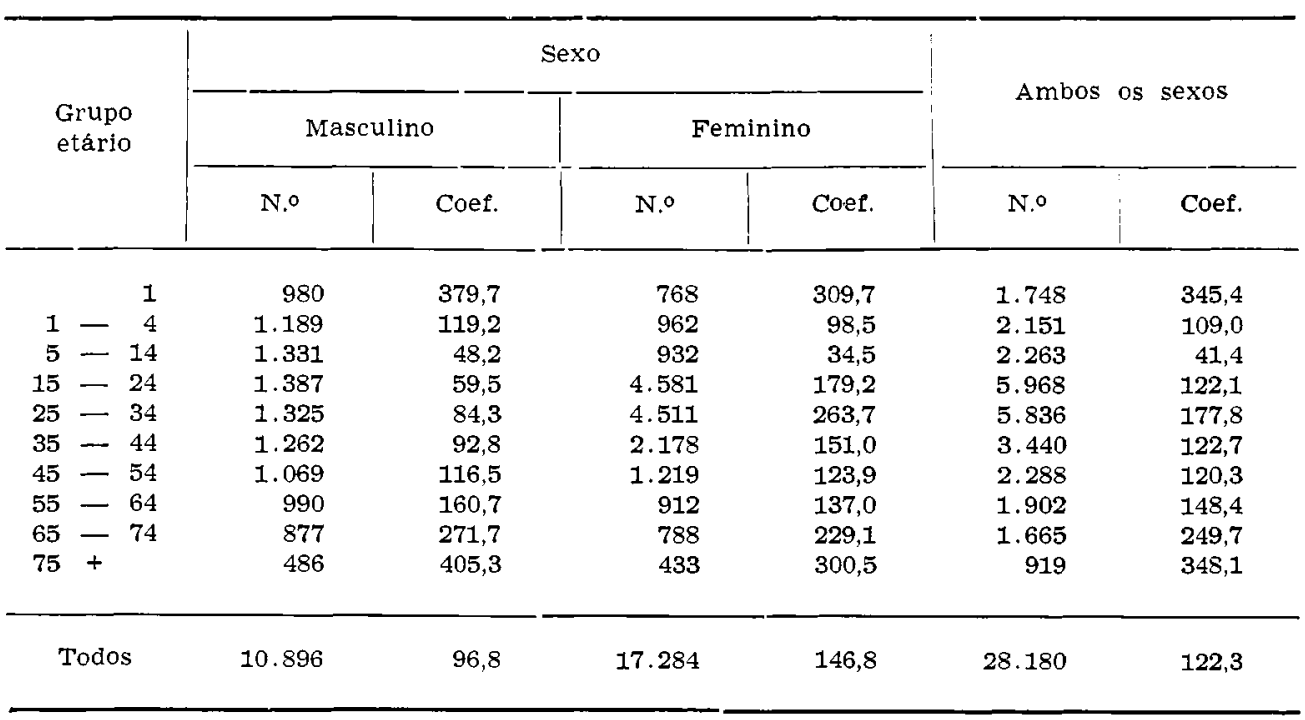

As internações procedentes de Ribeirão Preto (excluídos os recém-nascidos) foram: 10.896 internações de sexo masculino e 17.284 do sexo feminino, totalizando 28.180 internações (excluídos 54 casos de idade ignorada) (Tabela 1). A maioria das internaçôes de pacientes de Ribeirão Preto $(55,02 \%)$ ocorreu por conta do Instituto Nacional de Previdência Social, vindo a seguir a categoria de indigentes $(25,88 \%$ do total $) \mathrm{e}$ em terceiro lugar as internações de beneficiá- 
YAZLLE RoCHA, J. S. - Utilização de leitos hospitalares gerais em Ribeirão Preto, São Paulo (Brasil). Rer. Saúde pübl., S. Paulo, 9:477-93, 1975.

TABELA 2

Pacientes-dia, indice de hospitalização e duração média da internação da população de Ribeirão Preto, em hospitais gerais, segundo grupo etário e sexo, em 1972

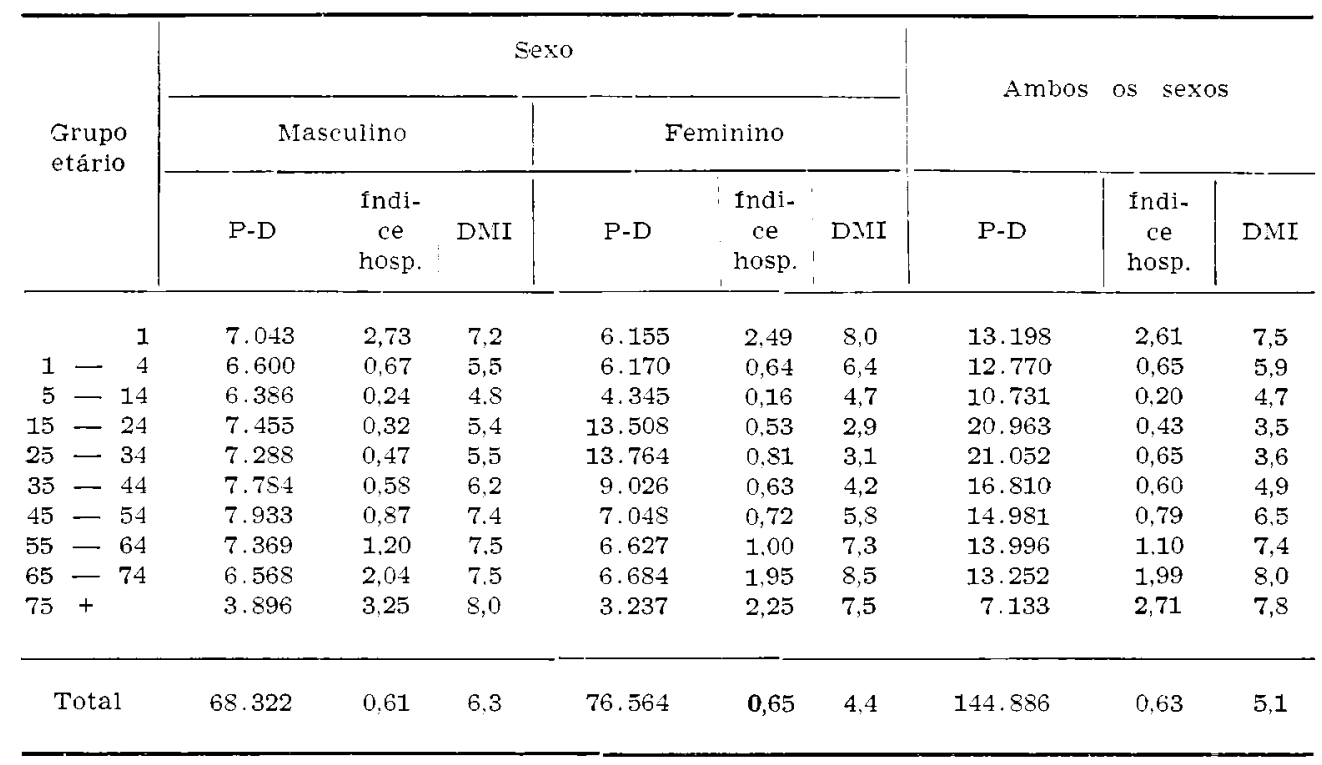

rios do Instituto de Assistência Médica dos Servidores Públicos do Estado (IAMSPE), do Serviço de Assistência e Seguro Social dos Municipários de Ribeirão Preto (SASSOM) e do Centro Social Regional do 3. ${ }^{\circ}$ Batalhão de Polícia $\left(3 .^{\circ} \mathrm{BP}\right)$, com $7.14 \%$ das internaçōes. A duração média das internaçôes foi diferente segundo a categoria da internação; as mais curtas foram as do Instituto Nacional de Previdência Social com 3.9 dias em média; em segundo lugar as "particulares" com 4.1 dias de duração média; as internaçôes mais demoradas foram da categoria de indigente com 7.8 dias de duração média.

Na Tabela 1 e Fig̣. 3 são apresentados os coeficientes de internação segundo grupo etário e sexo. $O$ coeficiente geral de internações (excluídos os recém-nascidos), para a população do município, foi de 122.3/1.000 hab.. sendo de 96.8 internaçoes/1.000 hab. para o sexo masculino e de $146.8 / 1.000$ hab. para o sexo feminino.
A Tabela 2 apresenta os pacientes-dia das internações de Ribeirão Preto e o índice de hospitalização para a população de Ribeirão Preto no ano de 1972, segundo grupo etário e sexo. 0 índice de hospitalização ${ }^{16}$ para a população geral foi de 0.63 dias/habitante-ano, sendo de 0.61 e de 0.65 dias/habitante-ano para os sexos masculino e feminino, respectiramente.

A Tabela 2 e Fig. 5 apresentam a duração média da internação segundo grupo etário e sexo lexcluídos os recém-nascidos). A duração média geral das internações foi de 5.1 dias, sendo de 6.3 dias para o sexo masculino e de 4,4 dias para o sexo feminino.

\section{3 - Avaliação do sistema de informações}

0 controle quantitativo das informações. efetuada rotineiramente pelo $\mathrm{CPDH}$, conferindo o número de folhas de egres- 
YAZLLE ROCHA, J. S. - Utilização de leitos hospitalares gerais em Ribeirão Preto, São Paulo (Brasil). Rev. Saude públ., S. Paulo, 9:477-93, 1975.

sos remetidas de cada hospital com o número de egressos de cada enfermaria, constatado no censo diário, funcionou satisfatoriamente em 1972. Em todos os casos de falta de folhas de egressos, foi possivel recuperar a informação.

Em relação à qualidade das informações processadas pelo $\mathrm{CPDH}$, definimos a consistência das mesmas como a percentagem da concordância entre as informações do cartão perfurado e as contidas no prontuário médico, do paciente correspondente. A idade do paciente em anos, mostrou concordância oscilando de hospital para hospital entre 86 e 99\%; como no nosso estudo utilizamos somente agrupamentos de idade, é de se esperar que a consistência dos dados por nós apresentados seja ainda maior. As outras condições pesquisadas (sexo, condição de saída e procedência) apresentaram consistência entre $90 \mathrm{e} 100 \%$.

\section{4 - DISCUSSÃO}

\section{1 - Sistema de informações}

Lima condição fundamental para a execução de estudos desta natureza é contar com bons sistemas de informações que preencham, entre outras, duas características básicas: cobertura e qualidade das informações. Nas rotinas do $\mathrm{CPDH}$, muita atenção é dada ao controle das informações e há um esquema para recuperar informaçōes, se necessário. Este controle nos assegura que não incorremos na perda quantitativa de informações de 1972.

Quanto à quantidade das informaçôes algumas considerações são necessárias. É preciso que o sistema de informação reflita o que é registrado no prontuário médico de cada paciente, no sentido de que a informação escrita na folha de egresso, codificada e perfurada, se mantenha em concordância com o que foi registrado pelo médico. 0 teste de consistência das informações por nós executado mostrou níveis de concordância entre 0 cartão perfurado e o prontuário médico acima de $90 \%$.

\section{2 - Condições de aplicação dos métodos}

Ao realizar o presente estudo, propusemo-nos a seguir uma metodologia que permitisse a aplicação de alguns dos métodos citados por Navarro ${ }^{19}$ (1969). Estes métodos são os baseados na utilização e na distribuição dos recursos (centrados nas facilidades e na população) e requerem que certas condições sejam satisfeitas (já referidas anteriormente). Em todos os casos é preciso contar com um sistema de informações que relacione as internações com a população em estudo. Realmente. em Ribeirão Preto há condiçōes para aplicar estes métodos ao estudo da utilização de leitos hospitalares gerais.

O município possui leitos hospitalares suficientes (5.25 leitos gerais/1.000 hab.) para, estudando sua utilização pela população local, encontrarmos a demanda destes serviços pela população. Este indice de leitos se encontra acima daqueles preconizados pela maioria dos autores brasileiros. Pode-se pensar que estes leitos sejam insuficientes para a população do município, visto que além da população local eles servem à região e até pacientes de outros estados. No entanto, incluindo os pacientes de fora do município, a ocupação destes leitos em 1972 foi de $70.71 \%$. isto é. mesmo atendendo à demanda regional ainda sobrou uma capacidade vaga nos hospitais de Ribeirão Preto.

Porém, quantidade de leitos apenas não é suficiente para obtermos a demanda; é preciso que existam relações geográficas e funcionais entre os recursos e a população (Burkens ${ }^{4}, 1966$; Navarro ${ }^{19}, 1969$; Yazlle Rocha e Fávero ${ }^{32}, 1972$ ); isto é, que os recursos sejam geográfica e financeiramente acessíveis e adequados às necessidades da população. Os hospitais do município de Ribeirão Preto estão situa- 
YAZLLE ROCHA, J. S. - Utilização de leitos hospitalares gerais em Ribelrão Preto, São Paulo (Brasil). Rev. Saúde públ., S. Paulo, 9:477-93, 1975.

dos no distrito do mesmo nome onde se encontra mais de $90 \%$ da população do município. Na cidade de Ribeirão Preto, os 7 hospitais gerais localizam-se em forma de anel em torno do centro comercial; todos os bairros da cidade são servidos por ônibus urbanos que chegam na sua maioria ao centro da cidade. Além disto, a Prefeitura local dispõe de 9 ambulâncias para o transporte de doentes que atendem principalmente os bairros periféricos e os distritos.

Existem em uso na cidade, vários sistemas de financiamento de hospitalizações, desde o "particular", seguros-saúde particulares (Cooperativa de Serviços Médicos, UNIMED), convênios empresas-hospitais, sistemas oficiais de cobertura previdenciária (INPS, IAMSPE), até os internados gratuitamente, cobertos por subvenções estatais ou pela caridade pública. Predominam amplamente as internações pelos sistemas previdenciários oficiais que, somados, financiam mais de $60 \%$ do total de internações do municipio. Seguem em freqüência as internações de "indigentes" que, somados aos anteriores, atingem quase $90 \%$ do total. Acreditamos que estes sistemas vigentes garantem a toda a população do município a atenção médico-hospitalar necessária.

Para que toda demanda de hospitalização seja satisfeita no município é preciso também que a atenção médico-hospitalar seja adequada às necessidades de saúde da população. Neste sentido, a cidade conta com um hospital-escola de renome, e existem também serviços modernos de pediatria, obstetrícia, cardiologia, ortopedia e traumatologia, neurologia, neurocirurgia e outros. Ribeirão Preto constitui, sem dúvida nenhuma, um centro médico regional ou de assistência terciária segundo critério citado por Navarro ${ }^{20}$ (1971). Muitos poucos casos devem ser encaminhados a outros centros porque aqui não encontram a assistência necessária: certas cirurgias cardíacas, quei- mados graves, alguns casos de neoplasias, que na maioria das vezes são pacientes da região que aqui vieram à procura da solução dos seus problemas. Os hospitais da cidade não guardam registro dos casos encaminhados a outros centros, mas podemos assegurar que não chegam a constituir um número significativo. Além disso, uma pessoa do município que por opção prefira atenção médica fora de Ribeirão Preto, terá de percorrer mais ou menos $200 \mathrm{~km}$ para encontrar um centro médico igual ou superior.

É preciso também que a população cuja demanda de atenção hospitalar está sendo pesquisada, seja bem definida e delimitada não mantendo continuidade com nenhuma população vizinha, o que poderia trazer dificuldades e também que esta população seja conhecida em algumas características.

A evidência direta da satisfação da demanda de hospitalização de uma dada população só pode ser obtida por levantamento ao nível da comunidade, a fim de se constatar se toda procura de hospitalização foi satisfeita. Entretanto as evidências indiretas acima apontadas permitem-nos assegurar que a utilização de leitos hospitalares gerais pela população do município constitui praticamente toda a demanda de hospitalização da população do município. Os fatos acima permitem-nos a aplicação dos métodos de investigação baseados na utilização e distribuição dos recursos.

\section{3 - Caracteristicas da utilização ou demanda de hospitalizações gerais no município de Ribeirão Preto, em 1972}

\subsection{1 - Coeficientes de internação}

Os coeficientes de internação para paises estudados tem apresentado amplas faixas de variação; San Martin ${ }^{27}$ (1968), no Chile, refere que estes valores giram 
YAZLLE ROCHA, J. S. - Utilização de leitos hospitalares gerals em Ribeirăo Preto, Săo Paulo (Brasil). Rev. Saúde puibl., S. Paulo, 9:477-93, 1975.

em torno de 10 internações/100 hab.; Anderson ${ }^{2}$ (1964) assinala grandes variações nos coeficientes dos EUA, da Grã-Bretanha e da Suécia, de 85 a 200 internaçōes/1.000 hab. A OPAS ${ }^{24}$ (1970) considera um mínimo desejável para a América Latina, coeficientes de 10 egressos/100 hab.-ano; refere que os coeficientes de egressos nesta região aumentaram no período de 1965-1968. Para países americanos, no ano de 1968, a OPAS apresenta os seguintes coeficientes de egressos/100 hab.:

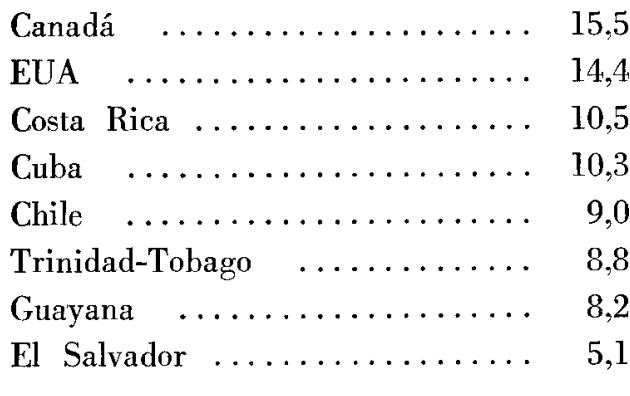

Nossos resultados mostram 12,2 internaçōes/100 hab. em 1972, próximo dos maiores valores dos países americanos em 1968.

Considerando os coeficientes de egressos por sexo e grupo etário, Witkins ${ }^{31}$ (1967) dá os seguintes valores estimados para hospitais "short-stay" no ano de 1965: para ambos os sexos, 153,4 egressos/1.000 hab.-ano, sendo para o sexo masculino de $123,5 / 1.000$ hab. e para o sexo feminino de 181,1/1.000 hab. Lembramos que os valores para Ribeirão Preto, em 1972, foram, respectivamente, 122,$3 ; 96,77$ e 146,81 internaçóes/1.000 hab. $O$ comportamento destes coeficientes, segundo as fontes acima, para Ribeirão Preto (1972) e EUA (1965) é apresentado nas Figs. 2 e 3.

Embora os valores dos coeficientes dos EUA, em média, sejam maiores que os encontrados em Ribeirão Preto, vemos que o comportamento dos mesmos é muito semelhante: para o sexo feminino, distribuição trimodal com picos de 0-1

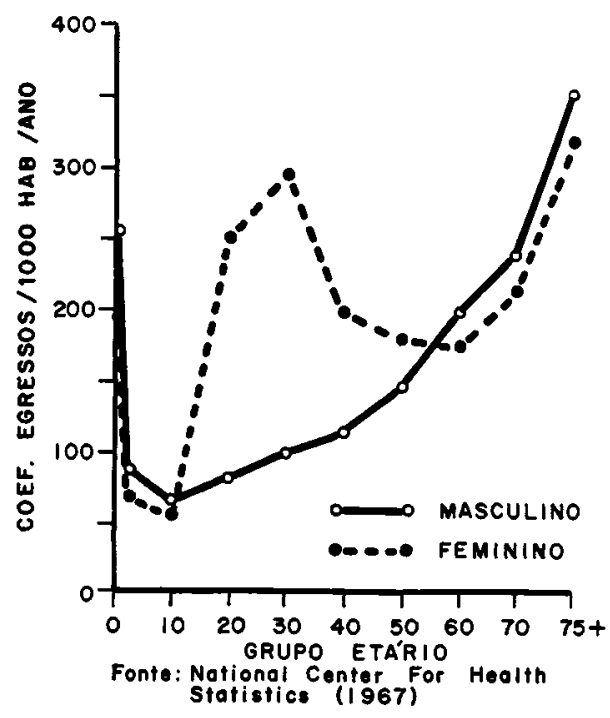

Fig. 2 - Coeficientes de egressos por 1.000 hab./ano, segundo grupo etário e sexo EUA, 1965.

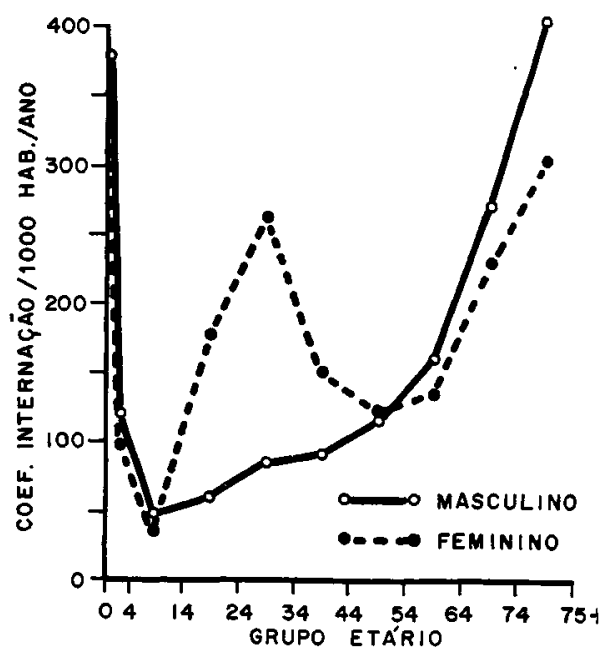

Fig. 3 - Coeficientes de internaçōes por 1.000 hab./ano, segundo grupo etário e sexo Ribeirão Preto, 1972.

ano, em torno dos 30 anos e elevação a partir dos 60 anos; para o sexo masculino, distribuição bimodal, com pico de 0-1 ano e maior elevação a partir dos 45 anos. 
YAZLLE RoCHA, J. S. - Utilização de leitos hospitalares gerais em Ribeirão Preto, São Paulo (Brasil). Rev. Saúde públ., S. Paulo, 9:477-93, 1975.

\section{3 .2 - Indice de hospitalização e duração média da internação}

O volume ou quantidade de recursos de atenção médico-hospitalar consumido pelas internações é medido pelo índice de hospitalização que nos dá os leitos-dia consumidos (pelos pacientes-dia de hospitalização). em relação ao totál da população (per-capita ou por 100 hab.). 0 volume de hospitalização depende do número de internações e da duração das mesmas; o número e a duração das internaçôes dependem, por sua vez, do tipo de população considerada (composição etária). do seu nírel de vida, do nível de saúde e dos tipos de problemas de saúde, da disponibilidade de atenção médica ambulatorial e hospitalar, entre outros fatores. Segundo a OPAS ${ }^{2+}$ (1970) no período de 1965-1968 observou-se redução na duração média da internação em países latino-americanos e no Canadá, enquanto que nos ELA aumentou no período considerado. Dessa publicação extraímos os dados a seguir que nos mostram os pacientes-dia por 100 hab. e a duração média de internação em hospitais gerais de países americanos em anos próximos de 1968.

\section{Pais}

Pacientes-

$D .11 I$ -dia/loo hab.

\begin{tabular}{lrr}
\hline Canadá & 168.3 & 10.8 \\
EUA & 134.7 & 9.4 \\
Cuba & 92.4 & 8.9 \\
Chile & 88.8 & 9.9 \\
Costa Rica & 73.9 & 7.1 \\
Guayana & 71.3 & 8.7 \\
Trinidad-Tobago & 63.3 & 7.2 \\
Jamáica & 55.6 & $9.7^{*}$ \\
Guatemala & 54.4 & 17.2 \\
\hline
\end{tabular}

* Iospitais do Governo somente.

Os dados correspondentes ao nosso estudo, para Ribeirão Preto. estão na Tabela 2. com 63 pacientes-dia/ 100 hab.-ano e 5.1 dias de duração média da interna- ção. Isto constitui um achado surpreendente. Enquanto no número de internaçōes por mil habitantes nos encontrávamos perto dos maiores valores observados entre estes países, o volume de hospitalizaçăo está próximo dos valores inferiores com uma duração média da internação inferior a destes países em 1968. Nestas condições o relativamente baixo índice de hospitalização decorre da surprendente baixa duração média das internações de Ribeirão Preto.

Witkins ${ }^{31}$ (1967) apresenta a duração média das internações para hospitais de "short-stay" em 1965. A DMI geral foi de 7,8 dias, sendo de 8.4 para o sexo masculino e de 7.5 dias para o sexo feminino. Suas variações segundo grupo etário e sexo são apresentadas em comparação com os resultados de Ribeirão Preto nas Figs. 4 e 5 .

Observamos nestas figuras que embora os valores estejam em níveis diferentes, o comportamento da DMI por grupo etário e sexo nos ELA e Ribeirão Preto é muito semelhante. Vemos que os grupos etários de internações mais demorados são. em média. crianças até um ano de idade. e principalmente adultos após a quinta década da vida. A duração média geral das internações vai depender muito da proporção destes grupos no total de internações. Não podemos esquecer aqui as variações observadas na DMI segundo a categoria da internação; a DMI mais curta de todas é a do Instituto Nacional de Previdência Social. que é o responsável também por mais da metade das internações no nosso município. Como se sabe, o INPS limita as internações clínicas (INPS, $1967^{11}$ ), cirúrgicas (INPS, $1967^{15}$ ) e obstétricas (INPS, $1966^{13}$ ) a respectivamente 20 dias (renováveis mediante justificação por escrito até o máximo de 60 dias), 3 dias para pequenas cirurgias, 6 dias para média cirurgia e 12 dias para grande cirurgia (a extensão destes períodos como as internações para tratamento pré-operatório ou para escla- 
YAZLLE ROCHA, J. S. - Utilização de leitos hospitalares gerais em Ribeirão Preto, São Paulo (Brasil). Rev. Saúde públ., S. Paulo, 9:477-93, 1975.

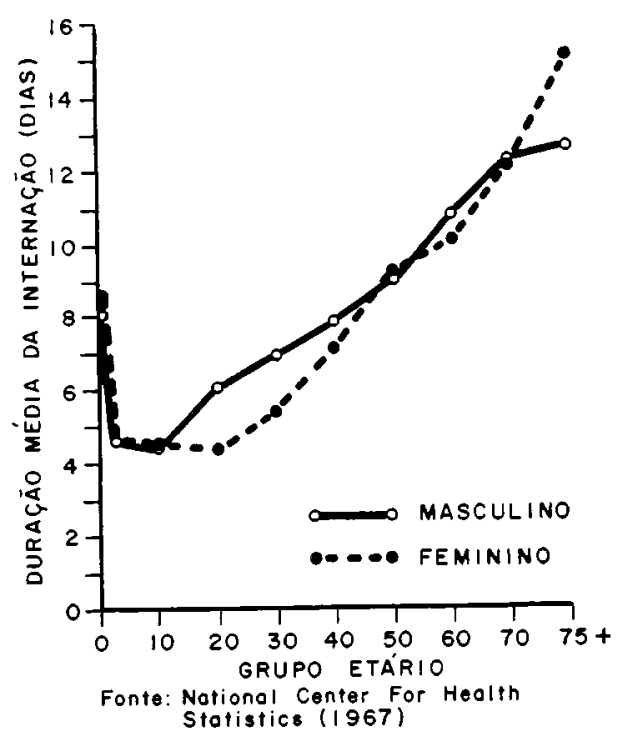

Fig. 4 - Duração média da internação, segundo grupo etário e sexo - EUA, 1965.

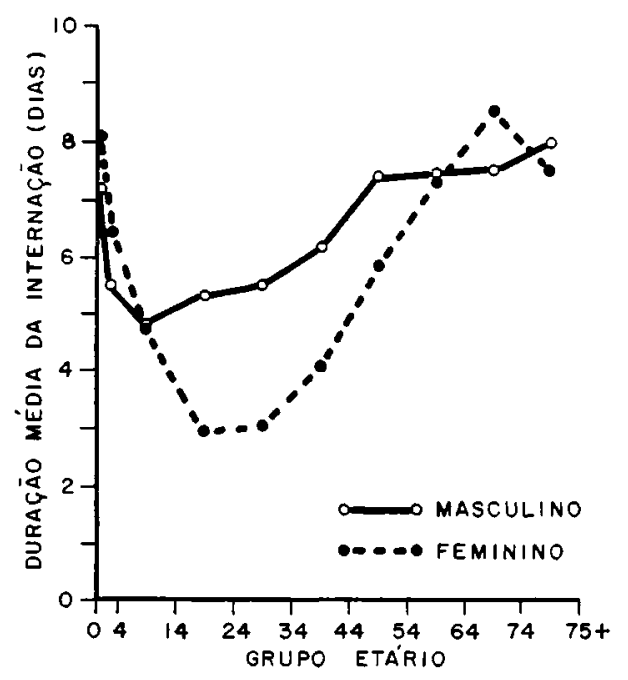

Fig. 5 - Duração média da internaçāo, segundo grupo etário e sexo - Ribeirão Preto, 1972.

recimento diagnóstico são permitidas mediante justificativa por escrito), 3 dias para parto espontâneo e até 5 dias para partos cirúrgicos (a permanência além deste prazo deverá ser justificada à autoridade local e por esta autorizada), e embora possa ser permitida a permanência por periodo maior que o estipulado, a obrigação de justificar por escrito deve ser uma das causas da curta DMI neste Instituto.

\subsection{3 - Utilização de leitos gerais}

0 total de pacientes-dia das internações de Ribeirão Preto, excluídos os recém-nascidos em hospitais gerais do município, em 1972, chegou a 145.058. Para produzir os leitos-dia suficientes para cobrir essa demanda, à percentagem de ocupação dos leitos hospitalares de Ribeirão Preto em 1972 (ou seja, de 70,71\%), são necessários 205.145 leitos-dia ao ano, ou 560 leitos gerais durante todo o ano, ou 2,43 leitos gerais/1.000 hab. Nos EUA, em 1965, segundo Witkins ${ }^{31}$, foram utilizados 3,3 leitos de "short-stay" por mil habitantes.

\section{4 - Algumas considerações sobre nossos achados}

A baixa utilização de leitos hospitalares gerais pela população do município de Ribeirão Preto decorre da curta duração média das internações. Esta, por sua vez, decorre de alguns fatores já discutidos anteriormente. Vimos pela Tabela 2 e Fig. 5 , que as durações médias de internações mais prolongadas ocorrem no primeiro ano de vida e após os 45 anos de idade; vimos também (Tabela 1, Fig. 3), que os coeficientes de internação para estes grupos etários são os maiores observados. Assim, é de se esperar que a utilização de leitos por uma população dependa, em grande parte, da proporção na população total de crianças até um ano de idade e de pessoas acima dos 45 anos.

Vimos que Ribeirão Preto apresenta os seguintes valores: $0-15$ anos: $34.50 \%$; 
YAZLLE ROCHA, J. S. - Utilização de leitos hospitalares gerais em Ribeirão Preto, São Paulo (Brasil). Rev. Saúde públ., S. Paulo, 9:477-93, 1975.

15-50 anos: $52,20 \%$; 50 anos ou mais: $13,30 \%$, ou seja, encontra-se mais próxima do tipo de população estacionária ou adulta (Sundbarg ${ }^{29}, 1965$ ) do que do tipo de população progressiva ou jovem (a pirâmide populacional brasileira aproxima-se mais do tipo progressiva ou jovem (Fundação HBGE ${ }^{11}$, 1973). Assim, a baixa utilização de leitos hospitalares em Ribeirão Preto poderia decorrer deste fato, o que é confirmado pelo seguinte:

\section{Percentagens no total de internações

$\begin{array}{ccc}\text { Grupos etários } & \begin{array}{c}\text { Ribeirão } \\ \text { Preto } \\ (1972)\end{array} & \begin{array}{c}\text { EUA } \\ (1965)\end{array} \\ 0-1 \text { ano } & 6,19 \% & 3,26 \%\end{array}$ \\ 45 anos ou mais $23,99 \% \quad 55,09 \%$}

Devemos destacar também as condições de saúde da população do município de Ribeirão Preto, as quais estão acima dos níveis da população geral brasileira (mortalidade geral $7,9 / 1.000$ hab., mortalidade infantil 43/1.000 nascidos vivos; mortalidade proporcional 50 anos ou mais de $55,23 \%$; grupo II segundo Swaroop ${ }^{30}$, 1964).

Este nível de saúde da população do município deve ser atribuido em primeiro lugar ao nível sócio-econômico da população e às condições de saneamento (água, esgotos, lixo) que não são comuns às cidades do país. Conta ainda com abundantes recursos para atenção médica-hospitalar ( 1 médico para 372 hab., 5,25 leitos gerais/1.000 hab. e outros já assinalados anteriormente).

Outro fator preponderante na DMI em Ribeirão Preto, é o sistema de hospitalização pelo Instituto Nacional de Previdência Social já comentado anteriormente.

Naturalmente, estas características da utilização de leitos hospitalares gerais pela população de Ribeirão Preto permanecerão enquanto os fatores que a condicionam persistirem. Podemos situar, assim, o município de Ribeirão Preto em uma fase especial da sua evolução histórica, caracterizada por certas condições demográficas, de infra-estrutura de serviços à população e de controle de alguns problemas de saúde mais comuns em regiốes menos desenvolvidas, em benefício da população presente.

Do ponto de vista das aplicações para o planejamento de assistência médica, nossas conclusóes se aplicam principalmente ao município de Ribeirão Preto, dentro da perspectiva por nós acima delineada. Nossas conclusões não podem ser extrapoladas diretamente a qualquer cidade ou região do país sem observar as restrições impostas pelas peculiaridades de cada local. Acreditamos também que nosso estudo será de utilidade ao chamar a atenção para alguns dos fatores envolvidos na assistência médico-hospitalar, principalmente para o fato de que a fase da evolução de uma comunidade pode determinar mudanças nos componentes demográficos e sociais, alterando profundamente (e até em sentidos inesperados, como a redução da utilização de leitos), a demanda de serviços pela população.

$$
5 \text { - CONCLUSOES }
$$

Através de um serviço de informática hospitalar é possível estudar a utilização de leitos hospitalares por uma determinada população.

A utilização de leitos hospitalares gerais pela população do município de $\mathrm{Ri}$ beirão Preto, constituiu praticamente toda a demanda de internações da população do município, no ano de 1972.

São características desta demanda:

- elevados coeficientes de internação (geral de 122,3 internaçōes/1.000 hab., sendo de $96,8 / 1.000$ hab. para o sexo masculino e de 146,8/1.000 hab. para o sexo feminino) ; 
YAZLLE ROCHA, J. S. - Utilização de leitos hospitalares gerais em Ribeirão Preto, São Paulo (Brasil). Rev. Saúde públ., S. Paulo, 9:477-93, 1975.

- baixo índice de hospitalização (geral de 0,63 dias/hab.-ano, sendo de 0,61 dias/hab.-ano para o sexo masculino e de $0,65 \mathrm{dias} / \mathrm{hal}$-ano para o sexo feminino;

- curta duração média das internações (geral de 5,1 dias, sendo de 6,3 dias para o sexo masculino e de 4,4 dias para o sexo feminino).
A composição etária da população e o sistema previdenciário oficial de hospitalização são importantes fatores na determinação de DMI em Ribeirão Preto.

À percentagem de ocupação dos leitos de $70,7 \%$, a demanda de internações gerais da população do município de Ribeirão Preto necessitou de 2,43 leitos gerais/1.000 hab., no ano de 1972 .

RSPU-B/286

Rocha, J. S. Y. - [On the use of general hospital beds in Ribeirão Preto (State of S. Paulo, Brazil)]. Rev. Saúde públ., S. Paulo, 9:477-93, 1975.

SUmMary: The city of Ribeirão Preto's general hospital beds demand in 1972 by the county's inhabitants was studied. Data from a hospital information center were used. The main characteristics of this demand were: high rate of in-patients, low hospitalization rate, short average length of permanence (caused by demographic factors and the hospitalization financing system) and finally 2.43 general hospital beds/1,000 inhabitants were used by the population that year.

UNITERMS: Hospitalization, demand. Beds (hospitals). Medical care.

\section{REFERENCIAS BIBLIOGRAFICAS}

1. ANDERSON, D. O. - Measurement of use demand - Uses of epidemiology in planning health services. [Paper presented at the International Scientific Meeting of the Internal Epidemiological Association, 6th, Belgrade, Yugoslávia, 1973].

2. ANDERSON, O. 'W. - Research in hospital use and expenditures. J. chron. Dis., 17:727-33, 1964.

3. BROOKS, G. H. et al. - A new development in predicting hospital bed needs. Int. Nurs. Rev., 11:33-9, 1964.

4. BURKENS, J. C. J. - The estimation of hospital bed requirements. Wld. Hosp., 2:110-3, 1966.

5. CASELlA, M. Z. F. - Determinação do número de leitos e respectivas especialidades para a cidade de São Paulo. Rev. paul. Hosp., $15(12): 29$ 37, 1967
6. FAVERO, M, et al. - Organização de um centro de informática hospitalar em nivel local. Rev. paut. Hosp., 21 (4) :151-7, 1973.

7. FELdSTEIN, P. J. \& GERMAN, J. J. Predicting hospital utilization: an evaluation of three approaches. Inquiry, 2:13, 1965.

8. FLISFISCH, H. - Planificación de hospitales $y$ otros servicios de salud. Washington, D.C., Organización Panamericana de la Salud, 1966. (OPAS - Publ. cient., 129).

9. FUNDACAO IBGE - Sinopse preliminar do censo demográfico: São Paulo. Rlo de Janeiro, 1971. (VIII Recenseamento Geral, 1970).

10. FUNDAÇO IBGE - Censo demográfico: São Paulo. Rio de Janeiro, 1971. (VIII Recenseamento Geral, 1970).

11. FUNDAÇAO IBGE - Anuário estatístico do Brasil. Rio de Janeiro, 1972. 
YAZLLE ROCHA, J. S. - Utilização de leitos hospitalares gerals em Ribeirão Preto, São Paulo (Brasil). Rev. Saúde puibl., S. Paulo, 9:477-93, 1975.

12. GONCCALVES, E. L. et al. - Assistência hospitalar no âmbito da previdencia social do Estado de São Paulo, Brasil. Rev. Saude públ., S. Paulo, 6: 35-43, 1972.

13. INSTITUTO NACIONAL DE PREVIDENCIA SOCIAL - Norma de serviço DNPS/PAPS n.o 2.5. Rio de Janeiro, 1966.

14 INSTITUTO NACIONAL DE PREVIDENCIA SOCIAL - Orientação de serviço n.o SAM 303.1. Rio de Janeiro, 1967.

15. INSTITUTO NACIONAL DE PREVIDENCIA SOCIAL - Orientação de serviço no SAM 302.1. Rio de Janeiro, 1967 .

16. LLEWELYN-DAVIES, R. \& MACAULAY, H. M. C. - Planificación y administración de hospitales. Washington, D.C., Organización Panamericana de la Salud, 1969. COPAS - Publ. cient., 191).

17. MARTINS, I. V. - O Governo do Estado no desenvolvimento da assistência hospitalar. Rev. paul. Hosp., 19 (11): 4-9, 1971.

18. MELLO, H. B. - Plano geral da rede médico-hospitalar de Brasilia. Rev. Serv. Saúde públ., Rio de Janeiro, 11:5-121, 1960

19. NAVARRO, V. - Planning for the distribution of personal health services. Publ. Hlth, Rep., 84:573-81, 1969.

20. NAVARRO, V. - Metodologia sobre planificación regional de servicios de salud. Estudio de un caso: Suécia. Cuad. Salud públ., Buenos Aires, $(6 / 7): 25-35,1970 / 71$.

21. NEWELL, D. J. - Problems of estimating the demand for hospital beds. J. chron. Dis., 17:749, 1964.

22. ORGANIZACION PANAMERICANA DE LA SALUD - Atención médica: bases para la formulación de una politica continental. Washington, D.C., 1962. (Publ. cient., 70).

23. ORGANIZACION PANAMERICANA DE LA SALUD - Administración hospitalaria en los programas de salud. Washington, D.C., 1967. (Publ. cient., 155).
24. ORGANIZACIÓN PANAMERICANA DE LA SALUD - Las condiciones de salud en las Américas; 1965-1968. Washington, D.C., 1970. (Publ. cient., 207).

25. PEDROSO, O. P. - Subsidios para um plano de desenvolvimento da assistência hospitalar no Estado de São Paulo. Rev. paul. Hosp., (8):9-28, 1954.

26. PUFFER, R. R. \& SERRANO, C. V. Caracteristicas de la mortalidad en la niñez. Washington, DC., Organización Panamericana de la Salud, 1973. (OPAS - Publ. clent., 262).

27. SAN MARTIN, H. - Salud y enfermedad: ecologia humana. Medicina preventiva y social. México, Prensa Médica Mexicana, 1968.

28. SAO PALlO (estado). Secretaria da Saúde. Levantamento de recursos para assistência médico-sanitária na 6. Região Administrativa. [Em vias de publicação].

29. SUNDBARG apud BERQLO, E. \& MILANESI, M. L. - Estatística vital. São Paulo, Faculdade de Higiene e Saúde Pública USP, 1965.

30. SWAROOP, S. - Estadística sanitaria. México. Fondo de Cultura Econôm!ca, 1964.

31. WITKINS, M. J. - Utilization of shortstay hospitals by characteristics of discharged patients. Washington, D.C., National Center for Health Statistics, 1967. (Vital and Health Statistics Series 13, n. 3).

32. YAZLLE ROCHA, J. S. \& FAVERO, M. Fatores que influenciam a demanda a um hospital regional. Med. CARL, Ribeirão Preto, 5:141, 1972.

33. YUNES, J. - Avaliação das necessidades de leitos hospitalares para o município de São Paulo e Região da Grande São Paulo nos anos de 1975 a 1990. Rev. paul. Hosp., $18(1): 3-8$, 1970.

Recebido para publicaço em 16/07/1975 Aprovado para publicą̧ão em 22/09/1975 